\title{
YOUNGER MATES AND THE JACOBIAN CONJECTURE
}

\author{
CHARLES CHING-AN CHENG, JAMES H. MCKAY, \\ AND STUART SUI-SHENG WANG
}

(Communicated by Wolmer V. Vasconcelos)

\begin{abstract}
Let $F, G \in \mathbb{C}[x, y]$. If the Jacobian determinant of $F$ and $G$ is 1 , then $G$ is said to be a Jacobian mate of $F$. If, in addition, $G$ has degree less than that of $F$, then $G$ is said to be a younger mate of $F$. In this paper, a necessary and sufficient condition is given for a polynomial to have a younger mate. This also gives rise to a formula for the younger mate if it exists. Furthermore, a conjecture concerning the existence of a younger mate is shown to be equivalent to the Jacobian conjecture.
\end{abstract}

Throughout this paper, $F$ and $G$ will be polynomials in $\mathbb{C}[x, y]$ where $\mathbb{C}$ denotes the field of complex numbers. We say that $F$ and $G$ satisfy the Jacobian hypothesis if their Jacobian determinant is one, i.e., $F_{x} G_{y}-F_{y} G_{x}=1$. In this case, we also say that $G$ is a Jacobian mate of $F$. Furthermore, if the $x$-degree (resp. $y$-degree, total degree) of $G$ is less than that of $F$, then $G$ is said to be a younger mate of $F$ relative to the $x$-degree (resp. $y$-degree, total degree). For instance, $x+y$ has younger mates $y$ and $-x$ relative to the $x$-degree and the $y$-degree, respectively, but has no younger mate relative to the total degree.

This paper was motivated by the Jacobian conjecture which asserts that if $F$ has a Jacobian mate $G$, then $(F, G)$ is an automorphism pair. In Section 1, it is shown that a younger mate is unique (up to an additive constant) and universal, i.e., if a Jacobian mate $G$ of $F$ exists, then any other mate of $F$ can be expressed as $G$ plus a polynomial in $F$. In Section 2, the problem of existence of a younger mate of $F$ is reduced to the case where $F$ is monic in both variables. In Section 3, a necessary and sufficient condition for the existence of a younger mate and a formula for a younger mate provided one exists are given. Finally, in Section 4, a conjecture concerning the existence of younger mates is formulated and shown to be equivalent to the Jacobian conjecture.

Received by the editors July 6, 1993 and, in revised form, January 18, 1994.

1991 Mathematics Subject Classification. Primary 13B25, 13F20, 14E09, 16W20.

Key words and phrases. Jacobian conjecture, Jacobian hypothesis, Jacobian mate, younger mate, automorphism, automorphism pair, Sylvester matrix, resultant, Newton polygon. 


\section{Properties of Jacobian mates}

Following [12, Theorem 33, p. 472], we shall use $\llbracket F, G \rrbracket$ to denote the Jacobian determinant. Note that $\mathbb{I}, \mathbb{1}$, as a function from $\mathbb{C}[x, y] \times \mathbb{C}[x, y]$ to $\mathbb{C}[x, y]$, is bilinear.

Theorem 1. Suppose $\llbracket F, G \rrbracket=1$ and $\llbracket F, K \rrbracket=0$ for $F, G, K$ in $\mathbb{C}[x, y]$. Then $K \in \mathbb{C}[F]$.

Proof. Since $\llbracket F, G \rrbracket=1, F$ and $G$ are algebraically independent over $\mathbb{C}$. Moreover, any $\mathbb{C}$-derivation on $\mathbb{C}[F, G]$ extends uniquely to a $\mathbb{C}$-derivation on $\mathbb{C}[x, y]$ by [13, Theorem 1, (1), (5), pp. 240-241]. In particular, $\frac{\partial}{\partial G}: \mathbb{C}[F, G]$ $\rightarrow \mathbb{C}[F, G]$ extends uniquely to $D: \mathbb{C}[x, y] \rightarrow \mathbb{C}[x, y]$. Indeed, the derivation $D$ is given by $D(H)=\llbracket F, H \rrbracket$ for $H \in \mathbb{C}[x, y]$ by [13, proof of $(5) \Longrightarrow(2)$ of Theorem 1, p. 241]. Then by [2, (1.5) Corollary, p. 74], $\mathbb{C}[F]=\operatorname{ker}(D)$. Since $D(K)=0$,

$$
K \in \operatorname{ker}(D)=\mathbb{C}[F] .
$$

Corollary 2 (Universality of Jacobian Mate). Suppose $\llbracket F, G \rrbracket=1$ and $\llbracket F, H \rrbracket$ $=1$ for $F, G, H$ in $C[x, y]$. Then $G-H \in \mathbb{C}[F]$.

Proof. Apply Theorem 1 with $K=G-H$.

Theorem 3 (Uniqueness of Younger Mate). If a younger mate relative to the $x$-degree (resp. $y$-degree, total degree) exists, then it is unique up to an additive constant.

Proof. Suppose both $G$ and $H$ are younger mates of $F$ relative to the $x$ degree. Then

$$
\llbracket F, G-H \rrbracket=\llbracket F, G \rrbracket-\llbracket F, H \rrbracket=1-1=0 .
$$

By Corollary 2, $G-H=\phi(F) \in \mathbb{C}[F]$. Since $\operatorname{deg}_{x}(G-H)<\operatorname{deg}_{x} F, \phi(F)$ is a constant. The proofs for $y$-degree and total degree are similar.

\section{REDUCTION}

In this section we shall reduce the problem of determining the existence of a younger mate of $F$ to the case where $F$ is monic in both $x$ and $y$. Recall that the Newton polygon for $F(x, y)$ is the convex hull of the origin together with the support of $F$.

Lemma 4. If $\operatorname{deg} F \geqslant 2, \operatorname{deg} G \geqslant 2$ and $\llbracket F, G \rrbracket=1$, then the Newton polygons of $F$ and $G$ are similar.

The first proof of this lemma was given by Oka [10, Lemma (6.1), p. 430]. The second proof was given by Applegate and Onishi [1, 14. Lemma, p. 217]. Because these proofs were incomplete, Nowicki and Nakai [8, Lemma B, p. 305] offered the third proof. However, as pointed out by L. Andrew Campbell in his review [Mathematical Reviews 89h:13007] of the article, this new proof still contained a gap. Moreover, M. Nagata also indicated an error of the new proof to the authors, so Nowicki and Nakai corrected this in [9].

However, we shall need the following stronger version which can be derived from Lemma 4 and its various proofs. 
Lemma 5. If $\operatorname{deg}_{x} F \geqslant 1, \operatorname{deg}_{y} F \geqslant 1, \operatorname{deg}_{x} G \geqslant 1, \operatorname{deg}_{y} G \geqslant 1$, and $\llbracket F, G \rrbracket=$ 1 , then the Newton polygons of $F$ and $G$ are similar with magnification factor equal to

$$
\frac{\operatorname{deg}_{x} F}{\operatorname{deg}_{x} G}=\frac{\operatorname{deg}_{y} F}{\operatorname{deg}_{y} G}=\frac{\operatorname{deg} F}{\operatorname{deg} G} .
$$

We have indicated in the beginning that a younger mate of $F$ relative to the $x$-degree (resp. $y$-degree) may not be a younger mate relative to the total degree. However, the next proposition shows that all three concepts coincide for a "nondegenerate" $F$.

Proposition 6. If $\operatorname{deg}_{x} F \geqslant 2, \operatorname{deg}_{y} F \geqslant 2$ and $\llbracket F, G \rrbracket=1$, then the following conditions are equivalent.

(1) $\operatorname{deg}_{x} G<\operatorname{deg}_{x} F$.

(2) $\operatorname{deg}_{y} G<\operatorname{deg}_{y} F$.

(3) $\operatorname{deg} G<\operatorname{deg} F$.

Proof. We first claim that $\operatorname{deg}_{x} G \geqslant 1$. Otherwise $G_{x}=0$, and the Jacobian hypothesis implies that $F_{x} \in \mathbb{C} \backslash\{0\}$. Hence $F=a x+p(y)$ for some nonzero constant $a$ and some polynomial $p(y) \in \mathbb{C}[y]$. This contradicts the assumption that $\operatorname{deg}_{x} F \geqslant 2$. Likewise $\operatorname{deg}_{y} G \geqslant 1$. Now the result follows from Lemma 5.

$F(x, y)$ is said to be monic in $x$ if either it is a polynomial in $y$ alone or it is of the form

$$
F(x, y)=x^{n}+p_{n-1}(y) x^{n-1}+\cdots+p_{1}(y) x+p_{0}(y)
$$

where every $p_{i}(y) \in \mathbb{C}[y]$ and $n \geqslant 1$. For any polynomial $F(x, y)$, there is an invertible linear substitution

$$
\left\{\begin{array}{l}
x=\alpha \bar{x}+\beta \bar{y} \\
y=\gamma \bar{x}+\delta \bar{y}
\end{array}\right.
$$

(so that $\alpha, \beta, \gamma, \delta \in \mathbb{C}$ and $\alpha \delta-\beta \gamma \neq 0$ ) such that

$$
\bar{F}(\bar{x}, \bar{y})=F(\alpha \bar{x}+\beta \bar{y}, \gamma \bar{x}+\delta \bar{y})
$$

is monic in $\bar{x}$ and $\bar{y}$ and $\operatorname{deg} F=\operatorname{deg}_{\bar{x}} \bar{F}=\operatorname{deg}_{\bar{y}} \bar{F}$ [5, (2.7), p. 5]. Note that the above substitution does not change the total degree nor the existence of a Jacobian mate of any polynomial. In the following we shall reserve $\bar{F}$ for the result of this transformation on the fixed polynomial $F$.

Proposition 7. Suppose $\operatorname{deg} F \geqslant 2$. Then the following conditions are equivalent.

(1) $F$ has a younger mate relative to the total degree.

(2) $\bar{F}$ has a younger mate relative to the $\bar{x}$-degree.

Proof. (1) $\Longrightarrow(2)$. Note that this implication does not need the hypothesis that $\operatorname{deg} F \geqslant 2$. Suppose $G$ is a younger mate of $F$ relative to the total degree. Let $\bar{G}(\bar{x}, \bar{y})=G(\alpha \bar{x}+\beta \bar{y}, \gamma \bar{x}+\delta \bar{y})$. Then, by the chain rule for Jacobians, $\frac{1}{\alpha \delta-\beta \gamma} \bar{G}$ is a Jacobian mate for $\bar{F}$. Moreover, $\operatorname{deg}_{\bar{x}} \bar{G} \leqslant \operatorname{deg} \bar{G}=\operatorname{deg} G<$ $\operatorname{deg} F=\operatorname{deg}_{\bar{x}} \bar{F}$. 
$(2) \Longrightarrow(1)$. Suppose $H$ is a younger mate of $\bar{F}$ relative to the $\bar{x}$-degree. Let $G(x, y)=H(\bar{\alpha} x+\bar{\beta} y, \bar{\gamma} x+\bar{\delta} y)$ where

$$
\left[\begin{array}{ll}
\bar{\alpha} & \bar{\beta} \\
\bar{\gamma} & \bar{\delta}
\end{array}\right]=\left[\begin{array}{ll}
\alpha & \beta \\
\gamma & \delta
\end{array}\right]^{-1}
$$

Then, by the chain rule for Jacobians, $(\alpha \delta-\beta \gamma) G$ is a Jacobian mate of $F$. Since $\operatorname{deg}_{\bar{x}} \bar{F}=\operatorname{deg}_{\bar{y}} \bar{F}=\operatorname{deg} F \geqslant 2$, we may apply Proposition 6 to $\bar{F}$ and $H$ to obtain $\operatorname{deg} H<\operatorname{deg} \bar{F}$. Thus $\operatorname{deg} G<\operatorname{deg} F$.

Combining Propositions 6 and 7, we see that if $\operatorname{deg}_{x} F \geqslant 2$ and $\operatorname{deg}_{y} F \geqslant 2$, then $F$ has a younger mate relative to any degree if and only if $\bar{F}$ has a younger mate relative to the $\bar{x}$-degree. In the next section we shall derive a necessary and sufficient condition for the latter.

\section{EXISTENCE OF YOUNGER MATES}

Lemma 8. Suppose that $\nu$ is a ring homomorphism from $R$ to $S$ where $R$ is a commutative ring and $S$ is an algebraically closed field. Let $H$ and $K$ be in $R[x]$. Then $\nu\left(\operatorname{Res}_{x}(H, K)\right)=0$ if and only if either $\operatorname{deg}_{x} \tilde{\nu}(H)<\operatorname{deg}_{x} H$ and $\operatorname{deg}_{x} \tilde{\nu}(K)<\operatorname{deg}_{x} K$, or $\tilde{\nu}(H)$ and $\tilde{\nu}(K)$ have a common linear factor in $S[x]$. Here $\tilde{\nu}: R[x] \rightarrow S[x]$ is the natural extension of $\nu$.

Proof. The nondegenerate case is proved in [11, Chapter 5, Section 8, p. 104, lines 2 to 5 ].

Proposition 9. Assume that $F$ is monic in $x$ with $x$-degree $\geqslant 1$. If $F$ has a Jacobian mate, then the resultant of $F_{x}$ and $F_{y}$ with respect to $x$ must be a nonzero constant.

Proof. To show that $r(y)=\operatorname{Res}_{x}\left(F_{x}, F_{y}\right)$ is a nonzero constant, it suffices to show that $r(y)$ has no roots. Suppose $r(y)$ has a root $y_{0}$ in $\mathbb{C}$. Applying Lemma 8 with $R=\mathbb{C}[y], S=\mathbb{C}$ and $\nu$ being the evaluation map at $y_{0}$, we see that $F_{x}\left(x, y_{0}\right)$ and $F_{y}\left(x, y_{0}\right)$ have a common linear factor in $\mathbb{C}[x]$ since

$$
\operatorname{deg}_{x} F_{x}\left(x, y_{0}\right)=\operatorname{deg}_{x} F_{x}(x, y)=n-1 .
$$

Let the common factor be $x-x_{0}$. Then $F_{x}\left(x_{0}, y_{0}\right)=F_{y}\left(x_{0}, y_{0}\right)=0$. However, setting $x=x_{0}$ and $y=y_{0}$ in the Jacobian hypothesis leads to $0=1$.

Suppose $F(x, y)$ is of the form (3.1) with $n \geqslant 2$ and $\operatorname{deg}_{y} F \geqslant 1$. Let $k$ be the largest $i$ such that $p_{i}(y)$ is a nonconstant. Since $\operatorname{deg}_{y} F \geqslant 1, k$ exists. Therefore

$$
\begin{aligned}
& F_{x}(x, y)=n x^{n-1}+(n-1) p_{n-1}(y) x^{n-2}+\cdots+p_{1}(y) \\
& F_{y}(x, y)=p_{k}^{\prime}(y) x^{k}+p_{k-1}^{\prime}(y) x^{k-1}+\cdots+p_{0}^{\prime}(y)
\end{aligned}
$$

Since $n \neq 0$ and $p_{k}^{\prime}(y) \neq 0, r(y)=\operatorname{Res}_{x}\left(F_{x}, F_{y}\right) \in \mathbb{C}[y]$ is the determinant of 
the following $(k+n-1) \times(k+n-1)$ Sylvester matrix $\mathbf{S}$ :

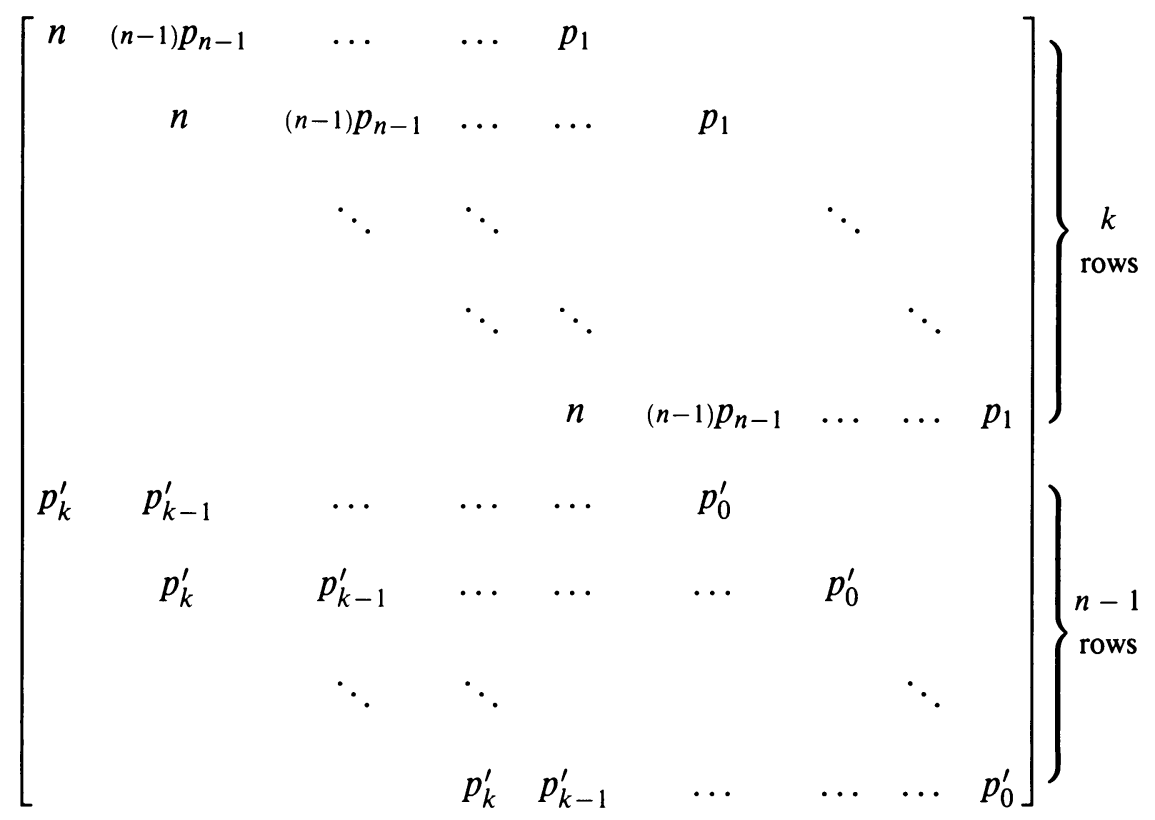

Let $\mathbf{S}^{\text {adj }}$ denote the adjoint of $\mathbf{S}$. If the bottom row of $\mathbf{S}^{\text {adj }}$ has entries $a_{1}(y)$, $a_{2}(y), \ldots, a_{k+n-1}(y)$, let

$$
\begin{aligned}
& \tilde{A}(x, y)=a_{1}(y) x^{k-1}+a_{2}(y) x^{k-2}+\cdots+a_{k}(y), \\
& \tilde{B}(x, y)=-\left(a_{k+1}(y) x^{n-2}+a_{k+2}(y) x^{n-3}+\cdots+a_{k+n-1}(y)\right) .
\end{aligned}
$$

Theorem 10. Suppose $F(x, y)$ is monic in $x$ with $\operatorname{deg}_{x} F \geqslant 2$ and $\operatorname{deg}_{y} F \geqslant 1$. Let $c=\operatorname{Res}_{x}\left(F_{x}, F_{y}\right)$. Then the following two conditions are equivalent.

(1) $F$ has a younger mate relative to the $x$-degree.

(2) $c \in \mathbb{C} \backslash\{0\}$ and $\tilde{A}_{x}=\tilde{B}_{y}$.

If these two conditions are satisfied, then the younger mate $G$ of $F$ relative to the $x$-degree is given by

$$
G=\frac{1}{c}\left(\int \tilde{B} d x+\int \tilde{A} d y-\iint \tilde{A}_{x} d y d x\right) .
$$

Proof. Multiplying both sides of the matrix equation

$$
\mathbf{S} \times\left[\begin{array}{c}
x^{k+n-2} \\
\vdots \\
x^{n-1} \\
x^{n-2} \\
\vdots \\
1
\end{array}\right]=\left[\begin{array}{c}
x^{k-1} F_{x} \\
\vdots \\
F_{x} \\
x^{n-2} F_{y} \\
\vdots \\
F_{y}
\end{array}\right]
$$


on the left by the adjoint of $\mathbf{S}$, we have

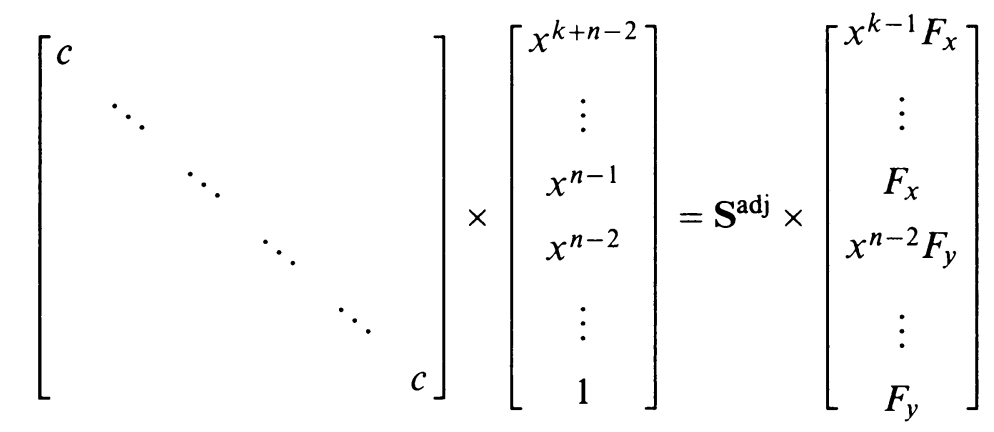

where $c=\operatorname{det} \mathbf{S}$. By comparing the bottom rows of both sides, we have

$$
\begin{aligned}
c= & a_{1}(y) x^{k-1} F_{x}+a_{2}(y) x^{k-2} F_{x}+\cdots+a_{k}(y) F_{x} \\
& \quad+a_{k+1}(y) x^{n-2} F_{y}+a_{k+2}(y) x^{n-3} F_{y}+\cdots+a_{k+n-1}(y) F_{y} \\
= & \tilde{A}(x, y) F_{x}-\tilde{B}(x, y) F_{y} .
\end{aligned}
$$

(1) $\Longrightarrow(2)$. If $F$ has a younger mate relative to the $x$-degree, then $c=$ $\operatorname{Res}_{x}\left(F_{x}, F_{y}\right)$ must be a nonzero constant by Proposition 9 . The last equation becomes

$$
1=A F_{x}-B F_{y}
$$

with $B=\frac{1}{c} \tilde{B}$ and $A=\frac{1}{c} \tilde{A}$. Now let $G$ be a younger mate of $F$ relative to the $x$-degree. Consider the matrix

$$
V=\left[\begin{array}{ccc}
B & B & A \\
F_{x} & F_{x} & F_{y} \\
G_{x} & G_{x} & G_{y}
\end{array}\right]
$$

Since the first two columns are identical, det $V=0$. On the other hand, expanding the determinant of $V$ according to the first column and using (4.1) and $\llbracket F, G \rrbracket=1$, we have $\operatorname{det} V=B \llbracket F, G \rrbracket-F_{x}\left(G_{y} B-G_{x} A\right)+G_{x}\left(F_{y} B-F_{x} A\right)=$ $B+F_{x}\left(G_{x} A-G_{y} B\right)-G_{x}$. Hence $G_{x}-B=F_{x}\left(G_{x} A-G_{y} B\right)$. Likewise, $G_{y}-A=F_{y}\left(G_{x} A-G_{y} B\right)$. So we have

$$
\left\{\begin{array}{l}
G_{x}-B=F_{x}\left(G_{x} A-G_{y} B\right), \\
G_{y}-A=F_{y}\left(G_{x} A-G_{y} B\right) .
\end{array}\right.
$$

We claim that $G_{x}-B=0$. Otherwise (4.2) implies that

$$
\operatorname{deg}_{x}\left(G_{x}-B\right)=\operatorname{deg}_{x} F_{x}+\operatorname{deg}_{x}\left(G_{x} A-G_{y} B\right) \geqslant \operatorname{deg}_{x} F_{x} .
$$

On the other hand, $\operatorname{deg}_{x} G<\operatorname{deg}_{x} F$. This, together with $\operatorname{deg}_{x} B \leqslant n-2<$ $n-1=\operatorname{deg}_{x} F_{x}$, shows that $\operatorname{deg}_{x}\left(G_{x}-B\right)<\operatorname{deg}_{x} F_{x}$, a contradiction. Therefore $G_{x}-B=0$. By the first equation of (4.2), we have $G_{x} A-G_{y} B=0$ since $F_{x} \neq 0$. Hence $G_{y}-A=0$ follows from the second equation of (4.2). Thus $G_{y x}-A_{x}=0$ and $G_{x y}-B_{y}=0$.

(2) $\Longrightarrow(1)$. Assume that $c$ is a nonzero constant and $\tilde{A}_{x}=\tilde{B}_{y}$. Let $B=\frac{1}{c} \tilde{B}$ and $A=\frac{1}{c} \tilde{A}$. Then $A_{x}=B_{y}$ and so there exists a polynomial $G(x, y) \in \mathbb{C}[x, y]$ such that $G_{x}=B$ and $G_{y}=A$. Then the $x$-degree of $G$ is at most $n-1$, and (4.1) becomes $1=\llbracket F, G \rrbracket$. 
Remark. If $\operatorname{deg}_{x} F=1, F$ does not have a younger mate relative to the $x$ degree.

\section{THE YOUNGER MATE CONJECTURE}

In this section we formulate the following conjecture and show that it is equivalent to the Jacobian conjecture.

The younger mate conjecture. Suppose that $F$ has a Jacobian mate and $\operatorname{deg} F \geqslant$ 2. Then there exists $G_{1}$ such that $\llbracket F, G_{1} \rrbracket=1$ and $\operatorname{deg} G_{1}<\operatorname{deg} F$. In other words, if $F$ has a Jacobian mate, then it has a younger mate relative to the total degree.

We say that $(F, G)$ is an automorphism pair if the $\mathbb{C}$-algebra homomorphism from $\mathbb{C}[x, y]$ to $\mathbb{C}[x, y]$ determined by $x \mapsto F$ and $y \mapsto G$ is an automorphism.

Lemma 11. Suppose $\llbracket F, G \rrbracket=1$ and $\llbracket F, H \rrbracket=1$. Then $(F, G)$ is an automorphism pair if and only if $(F, H)$ is also an automorphism pair.

Proof. By Corollary $2,(F, G)=(F, H+\phi(F))=(x, y+\phi(x)) \circ(F, H)$. Now the result follows from the fact that $(x, y+\phi(x))$ is an automorphism pair.

Lemma 12. If $(F, G)$ is an automorphism pair with $\operatorname{deg} F \leqslant \operatorname{deg} G$, then $\operatorname{deg} F$ divides $\operatorname{deg} G$.

Proof. For an elementary proof, as well as a historical account, see [7].

Lemma 13 (Dixmier [4, 2.7. Lemme, p. 215]). If $\llbracket f, g \rrbracket=0$ where $f, g \in$ $\mathbb{C}[x, y]$ are nonzero and homogeneous, then $\frac{f^{\operatorname{deg} g}}{g^{\operatorname{deg} f}} \in \mathbb{C} \backslash\{0\}$.

Corollary 14. Suppose $(F, G)$ is an automorphism pair with $\operatorname{deg} F \leqslant \operatorname{deg} G$. Then there exists $\lambda \in \mathbb{C} \backslash\{0\}$ such that $\operatorname{deg} G_{1}<\operatorname{deg} G$ and $G_{1}=G-$ $\lambda F^{\operatorname{deg} G / \operatorname{deg} F}$.

Proof. The case in which $\operatorname{deg} F=\operatorname{deg} G=1$ is easy to see, so we only consider the case where $\operatorname{deg} G \geqslant 2$. Let $F^{+}$and $G^{+}$be the highest homogeneous part of $F$ and $G$ respectively. Since $(F, G)$ is an automorphism pair, $\llbracket F, G \rrbracket \in \mathbb{C} \backslash\{0\}$ by the chain rule for Jacobians. Using the biadditivity of the Jacobian and counting degrees, we obtain $\llbracket F^{+}, G^{+} \rrbracket=0$. By Lemma 13, $\left(F^{+}\right)^{\operatorname{deg} G} /\left(G^{+}\right)^{\operatorname{deg} F} \in \mathbb{C} \backslash\{0\}$. Since $\operatorname{deg} F$ divides $\operatorname{deg} G$ by Lemma 12 , it

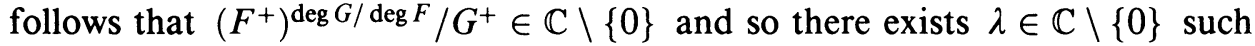
that $G^{+}=\lambda\left(F^{+}\right)^{\operatorname{deg} G / \operatorname{deg} F}$. Hence the result follows.

Lemma 15. Suppose $\llbracket F, G \rrbracket=1$ with $\operatorname{deg} G=1$. Then $(F, G)$ is an automorphism pair.

Proof. After a suitable change of coordinates, we may assume that $G=y$. Then $\llbracket F, G \rrbracket=1$ implies that $F_{x}=1$, i.e., $F=x+p(y)$ for some $p(y) \in \mathbb{C}[y]$.

Theorem 16. The younger mate conjecture is equivalent to the Jacobian conjecture.

Proof. $\Longrightarrow$ : Suppose the younger mate conjecture is true, and suppose $\llbracket F, G \rrbracket$ $=1$. Then there exists $G_{1}$ such that $\llbracket F, G_{1} \rrbracket=1$ and $\operatorname{deg} G_{1}<\operatorname{deg} F$. Hence $\llbracket G_{1},-F \rrbracket=1$ and, by the younger mate conjecture, there exists $G_{2}$ such that 
$\llbracket G_{1}, G_{2} \rrbracket=1$ and $\operatorname{deg} G_{2}<\operatorname{deg} G_{1}$ provided that $\operatorname{deg} G_{1} \geqslant 2$. Repeating this process, we obtain a decreasing sequence

$$
\operatorname{deg} F>\operatorname{deg} G_{1}>\operatorname{deg} G_{2}>\cdots
$$

such that

$$
\llbracket G_{i}, G_{i+1} \rrbracket=1
$$

for $i \geqslant 0$ where $F=G_{0}$. This sequence must stop, say, at $G_{s}$. Then $\operatorname{deg} G_{s} \leqslant 1$ and hence $\operatorname{deg} G_{s}=1$ by $\llbracket G_{s-1}, G_{s} \rrbracket=1$. By Lemma $11,(F, G)$ is an automorphism pair if and only if $\left(F, G_{1}\right)$ is. Similarly, as $\llbracket G_{i+1}, G_{i+2} \rrbracket$ $=\llbracket G_{i+1},-G_{i} \rrbracket=1,\left(G_{i+1}, G_{i+2}\right)$ is an automorphism pair if and only if $\left(G_{i+1},-G_{i}\right)$ is. The latter is equivalent to saying that $\left(G_{i}, G_{i+1}\right)$ is an automorphism pair. So $(F, G)$ is an automorphism pair if and only if $\left(G_{s-1}, G_{s}\right)$ is. But $\left(G_{s-1}, G_{s}\right)$ is an automorphism pair by Lemma 15 .

$\Longleftarrow:$ Suppose the Jacobian conjecture is true, and suppose that $\llbracket F, G \rrbracket=1$ with $\operatorname{deg} F \geqslant 2$. We may assume that $\operatorname{deg} F \leqslant \operatorname{deg} G$ for otherwise we are done. Then the Jacobian conjecture implies that $(F, G)$ is an automorphism pair. By Corollary 14, there exists $G_{1}$ such that $\operatorname{deg} G_{1}<\operatorname{deg} G$ and $\llbracket F, G_{1} \rrbracket=1$. If $\operatorname{deg} G_{1} \geqslant \operatorname{deg} F$, then using Corollary 14 again, we obtain $G_{2}$ such that $\operatorname{deg} G_{2}<\operatorname{deg} G$ and $\llbracket F, G_{2} \rrbracket=1$. Repeat this process to create a decreasing sequence

such that

$$
\operatorname{deg} G>\operatorname{deg} G_{1}>\operatorname{deg} G_{2}>\cdots
$$

$$
\llbracket F, G_{i} \rrbracket=1
$$

for all $i \geqslant 1$. The sequence must stop, say, at $G_{s}$. Then $\llbracket F, G_{s} \rrbracket=1$ and $\operatorname{deg} G_{s}<\operatorname{deg} F$.

Remark. If the conclusion $\operatorname{deg} G_{1}<\operatorname{deg} F$ in the younger mate conjecture is replaced by $\operatorname{deg} G_{1}<\operatorname{deg} G$, then it becomes false (although it is easy to see that the result is stronger than before). For example, if $F=y+\left(x+y^{2}\right)^{2}$ and $G=x+y^{2}$, then such $G_{1}$ does not exist: As $\operatorname{deg} G_{1}<\operatorname{deg} G=2$, we may write $G_{1}=\alpha x+\beta y+s$ where $\alpha, \beta, s \in \mathbb{C}$; then $\llbracket F, G_{1} \rrbracket=-\alpha+2\left(x+y^{2}\right)(\beta-2 \alpha y) \neq$ 1 .

Addendum. Yosef Stein has also announced some conditions for existence of a Jacobian mate in his talk entitled "The Jacobian problem in two variables as a system of ordinary differential equations" in the International Conference on Polynomial Automorphisms and Related Topics held in CIRM (Centre International de Rencontres Mathématiques), Luminy, Marseille, France in October 1992.

\section{REFERENCES}

1. H. Applegate and H. Onishi, The Jacobian conjecture in two variables, J. Pure Appl. Algebra 37 (1985), 215-227. (MR 87b:14005. Zbl.571.13005)

2. H. Bass, Differential structure of étale extensions of polynomial algebras, Commutative Algebra: Proceedings of a Microprogram (June 15-July 2, 1987, MSRI, Berkeley, CA) (M. Hochster, C. Huneke, and J. D. Sally, eds.), Math. Sci. Res. Inst. Publ., vol. 15, Springer-Verlag, New York, 1989, pp. 69-108.

3. Z. Charzyński and P. Skibiński, A criterion for explicit dependence of polynomials, Bull. Soc. Sci. Lett. Lódź 37 (1987), 1-3. (MR 89g:120007. Zbl.653.26014) 
4. J. Dixmier, Sur les algèbres de Weyl, Bull. Soc. Math. France 96 (1968), 209-242. (MR 39\#4224. Zbl.165,49)

5. S. Lefschetz, Algebraic geometry, Princeton Math. Ser., vol. 18, Princeton Univ. Press, Princeton, NJ, 1953. (MR 15-150)

6. J. H. McKay and S. S.-S. Wang, An inversion formula for two polynomials in two variables, J. Pure Appl. Algebra 40 (1986), 245-257. (MR 87j:12003. Zbl.622.13003)

7. An elementary proof of the automorphism theorem for the polynomial ring in two variables, J. Pure Appl. Algebra 52 (1988), 91-102. (MR 89k:14017. Zbl.656.13002)

8. A. Nowicki and Y. Nakai, On Applegate-Onishi's lemmas, J. Pure Appl. Algebra 51 (1988), 305-310. (MR 89h:13007. Zbl.661.12009)

9. __ Correction to "On Applegate-Onishi's lemma", J. Pure Appl. Algebra 58 (1989), 101. (MR 90b:13007. Zbl.676.12006)

10. M. Oka, On the boundary obstructions to the Jacobian problem, Kōdai Math. J. 6 (1983), 419-433. (MR 85m:14023. Zbl.526.13013)

11. B. L. van der Waerden, Algebra, Vol. 1, Ungar, New York, 1970 (translated from the 1966 $7^{\text {th }}$ German edition). (MR $41 \# 8187 a$ )

12. S. S.-S. Wang, A Jacobian criterion for separability, J. Algebra 65 (1980), 453-494. (MR 83e:14010. Zbl.471.13005)

13. __ Extension of derivations, J. Algebra 65 (1980), 453-494. (MR 82e:13005. Zbl.458. 13001)

Department of Mathematical Sciences, Oakland University, Rochester, Michigan 48309-4401

E-mail address: cheng@vela.acs. oakland.edu

E-mail address: mckay@vela.acs.oakland.edu

E-mail address: swang@vela.acs.oakland.edu 